\title{
COMBINING ABILITY AND HETEROSIS IN TOMATO UNDER HIGH TEMPERATURE CONDITIONS
}

\author{
Entsar M. I. Abo-Hamda \\ Vegetables Breeding Dept., Hort. Res. Inst., Agricultural Research Center (ARC), Giza, Egypt. \\ Received: Apr. 13, 2017 \\ Accepted: Apr. 27, 2017
}

\begin{abstract}
This investigation was conducted during 2014 and 2015 at Kalubia governorate to develop promising hybrids of tomato (Solanum lycopersicum L.) for yield and fruit quality characters under high temperature stress in Egypt using Line $x$ Tester matting design. All studied traits, i.e., fruit set, total yield, marketable yield, average fruit weight, fruit shape index, fruit firmness, number of locules, total soluble solids (TSS\%), vitamin C content and titratable acidity have closer values of $\sigma^{2} g$ and $\sigma^{2} p$, meanwhile, the G.C.V. and P.C.V.\% which was confirmed by the estimated G.C.V./P.C.V. ratios and high broad sense heritability (BSH) values suggest less effect of environment and the large portion of $\sigma^{2} p$ was due to the $\sigma^{2} g$ on these traits, except number of locules and titratable acidity traits which was affected by both genetic and environmental factors. The ratio of $\sigma^{2} G C A / \sigma^{2} S C A$ were found less than unity $(<1)$ indicating the preponderance of non additive gene actions over the additive ones for all the studied traits. The prevalence of the non-additive variance suggested heterosis breeding approach is effective way for improvement of these traits. Most of the traits exhibited significant hybrid vigor for some of crosses based on the better-parent. The lines Ent 5 and Ent 17 and the tester TLB 111 showed maximum positive GCA effects for most of the important traits. So, these parents could be successfully used in future for breeding programs. Among all hybrids, Ent $3 \times 99 S-C-39$, Ent $5 \times 99 S-C-39$ and Ent $31 \times$ TLB 111 exhibited significant SCA effects for both total and marketable yield characters under heat stress. So, these hybrids could be used in future for breeding to these traits.
\end{abstract}

Key words: Tomato, Solanum lycopersicum, Heat tolerance, Heterosis, Combining ability, heritability, GCA, SCA.

\section{INTRODUCTION}

Tomato (Solanum lycopersicum L.) is one of the most important vegetable crops grown throughout the world because of its wider adaptability, high yielding potential and suitability for uses as salad, cooked or processed into several preferred products like ketchup, juice, puree, sauce and whole canned fruit. In Egypt, shortage of tomatoes production is common due to high temperatures in late summer season. Tomato is adapted to a wide range of climates while fruit set is limited to a somewhat narrow range. High temperature during reproductive development caused significant increment in flower drop and significant decrease in fruit set (Berry et al 1988) and consequently fruit yield decreased to a great extent. At high temperature, the reproductive part of the flower is adversely affected. Stigma tube elongation, poor pollen germination, poor pollen tube growth and carbohydrate stress are the main reasons for poor fruit set at high temperature in tomato. El-Ahmadi and Stevens (1979) also said that fruit setting in tomato is interrupted at temperature above $26{ }^{\circ} \mathrm{C}$ and $20{ }^{\circ} \mathrm{C}$ day/night and is often completely arrested at temperature above $38 / 27{ }^{\circ} \mathrm{C}$ day/night. However, Metwally et al (1988) indicated that for optimum fruit setting, tomato plants require night temperature of $14-20^{\circ} \mathrm{C}$ and day temperature of $25-30^{\circ} \mathrm{C}$. When night or day temperature was higher or lower than this rang fruit setting was reduced or completely terminated. However, temperature higher than $34 / 20^{\circ} \mathrm{C}$ (day/night) or a period of 4 
hours at $40^{\circ} \mathrm{C}$ cause blossom drop in most cultivars.

The knowledge of genetic structure and mode of inheritance of different characters helps breeders to employ suitable breeding methodology for their improvement. In any breeding programme, the proper choice of parents based on their combining ability is a prerequisite. Combining ability is an important in plant breeding since it provides information for selection of parents and also provides information regarding nature of gene actions. In this direction, the concept of general (GCA) and specific (SCA) combining abilities helps the breeder to decide upon the choice of parents for hybridization and also gives information on gene action, which helps in understanding the nature of inheritance of the characters (Sprague and Tatum 1942). Griffing (1956) stated that GCA effects were due to additive type of gene action and SCA effects were due to non-additive (dominant or epistatic) gene action. In this context, Line $\times$ Tester mating design proposed by Kempthrone (1957) helps the breeders by providing information on the combining ability status of genotypes (parents and hybrids) used and also on the nature of gene action involved.

In plant breeding, tomato hybrids had contributed a lot in terms of production. The estimation of heterosis for yield and fruit quality characters is useful to judge the best hybrid combination for exploitation of superior hybrids. Heterosis over better parent on tomato was reported for some traits, i. e., average fruit weight, TSS and total yield by Mondal et al (2009), for fruit set, TSS, firmness, total yield, by Shalaby (2012), for fruit weight, fruit yield per plant, fruit firmness and total soluble solids, by Saeed et al (2014) and for fruit firmness, TSS, average fruit weight, yield per plant by Khalil et al (2015). However, heterosis was found absent for average fruit weight (Shalaby 2012), for fruit yield, TSS, and fruit firmness (Kalenahalli and Gowda 2013) and for fruit set trait (Khalil et al 2015).
Several studies of combining ability for yield and fruit quality characters are available in tomato. The additive variance was larger than non-additive variance and the ratio of additive variance and non additive genetic variance is more than unity, establishing the predominance of additive gene action in the inheritance of the traits average fruit weight, total yield per plant, TSS, ascorbic acid, titratable acidity (Kumar et al 2013), average fruit weight (Shankar et al 2013), total yield (Saeed et al (2014) TSS, fruit acidity and ascorbic acid (Dagade et al 2015) and fruit firmness (Khalil et al 2015). Hence, significant advancement could be achieved in the segregating generations using simple selection procedures or conventional breeding methods such as pedigree and bulk selection, which are useful for accumulation of desirable genes for these traits.

However, non-additive genetic variance had greater estimates than additive genetic variance and the ratio of additive variance and non additive genetic variance is less than unity, establishing the predominance of non additive gene action in the inheritance of the traits total soluble solids and tritable acidity (Mondal et al 2009), yield per plant (Dagade et al 2015 and Shankar et al 2013), total yield, average fruit weight, fruit firmness, TSS, ascorbic acid (Kansouh and Zakher 2011), total soluble solids, ascorbic acid, acidity, average fruit weight, fruit yield per plant (Katkar et al 2012), TSS, fruit firmness, yield/plant (Kalenahalli and Gowda 2013), fruit weight, fruit firmness and total soluble solids (Saeed et al (2014) and TSS, fruit set, average fruit weight and yield per plant (Khalil et al 2015). The presence of non-additive gene action suggests that heterosis breeding method is effective for improvement of these traits.

Low values of difference between genotypic coefficient of variation (GCV\%) and phenotypic (PCV\%) coefficients of variations, as well as, high broad sense heritability $(\mathrm{BSH})$ for the traits total yield, 


\section{Combining ability and heterosis in tomato under high temperature conditions.}

average fruit weight, fruit firmness, TSS, acidity and ascorbic acid contents were observed by Kansouh and Zakher (2011) and Salib (2012).

The maximum day and minimum night temperatures in Egypt are frequently getting higher than $30{ }^{\circ} \mathrm{C}$ and $20^{\circ} \mathrm{C}$, respectively, during summer season. Therefore, the objective of the present study was to identify breeding lines/varieties having good combining ability effects and best cross combinations for developing promising hybrids with yield and fruit quality characters under high temperature stress using Line $\mathrm{x}$ Tester mating design.

\section{MATERIALS AND METHODS}

This investigation was carried out during the period from 2014 to 2015. Thirteen tomato pure lines were evaluated under high temperatures stress during 2014 in late summer season to insure high degree of homozygosity of each parent before crossing. These pure lines were Ent $2\left(L_{1}\right)$, Ent $3\left(L_{2}\right)$, Ent $5\left(L_{3}\right)$, Ent $8\left(L_{4}\right)$, Ent $9\left(L_{5}\right)$, Ent $12\left(L_{6}\right)$, Ent $17\left(L_{7}\right)$, Ent $28\left(L_{8}\right)$, Ent 31 $\left(L_{9}\right)$ and Ent $37\left(L_{10}\right)$ which was used as females (Lines) and TLB $111\left(\mathrm{~T}_{1}\right)$, TLB 182$1\left(\mathrm{~T}_{2}\right)$ and 99S-C-39 $\left(\mathrm{T}_{3}\right)$ which was used as males (testers). All these genotypes were produced from previous tomato breeding program by selfing and selection during 6 generations at Vegetable Breeding Dep., Hort. Res. Inst., Agric. Res. Center, Egypt (Abo-Hamda 2004), except the genotypes TLB 111 and TLB 182-1 which were kindly collected from Asian Vegetable Research and Development centre (AVRDC), Taiwan. The females were chosen for genetic studies based on their performance of yield and other desirable economic characters, viz., yield, yield components and fruit quality. Males were chosen as heat resistance sources. Selfing and crosses were made manually using the standard procedure of hand emasculation and pollination in the greenhouse at Kaha Vegetable Research Farm, Kalubia Governorate during the fall season of 2014. Each female line was crossed with the three other males (testers).

Then, all genotypes (13 parents and 30 $F_{1}$ hybrids) were evaluated in the open field under high temperature conditions at private farm, Kalubia Governorate during late summer of 2015 season. The nursery of each accession was transplanted in a field in three replicates following randomized complete block design layout. Each genotype was grown on one ridge. The seedlings were planted in rows having 10 plants per row keeping row-to-row and plantto-plant distances of $80 \mathrm{~cm}$ and $40 \mathrm{~cm}$, respectively. Land preparation and field practices were applied according to recommendations of the Egyptian Ministry of Agriculture. Seeding and transplanting dates were at April $3^{\text {th }}$ and May $18^{\text {th }}, 2015$, respectively. Averages of temperatures during the growing evaluation season of the study at Kalubia governorate were 25/15.2, 29/21.2, 31/24.1, 33/23.5 and $35 / 24.1^{\circ} \mathrm{C}$ day/night in April, May, June, July and August, respectively (Central Laboratory for Agricultural Climatic, Ministry of Agriculture and Land Reclamation, Egypt).

Data were recorded on 5 randomly chosen plants/plot for the studied traits: fruit set\% which was calculated as the number of fruits set compared with the total number of flowers on the first 3 clusters, total yield (ton/feddan and feddan $=4200 \quad \mathrm{~m}^{2}$ ), marketable yield (ton/feddan), average fruit weight (g), fruit shape index which calculated as the ratio of fruit length to fruit width and oval fruit shape is usually considered for a ratio greater than 1.2, round shape for a ratio of 0.95-1.2 and oblate shape for a ratio less than 0.95 (Yeager 1937), fruit firmness $\left(\mathrm{g} / \mathrm{cm}^{2}\right)$, number of locules/fruit, total soluble solids (TSS\%), vitamin C content (mg/100 g fresh fruit) and titratable acidity (mg citric acid/100 $\mathrm{g}$ fresh fruit).

The data for all traits were analyzed following Kempthorne (1957). Heterosis over 
better parent was calculated as percent according to Sinha and Khanna (1975).

Heterosis $(\%)=\left[\left(\overline{F_{1}}-\overline{B P}\right) / \overline{B P}\right] * 100$

Where, $\overline{F_{1}}=$ mean performance of cross

and $\overline{B P}=$ mean performance of better parent

\section{RESULTS AND DISCUSSION \\ 1 - Analysis of variance and mean square values for the mating design Line $x$ Tester:}

Data of Table 1 show that the replications had no-significant differences, however, the mean squares of genotypes were significant for all studied traits indicating the presence of adequate genetic variability and the genetic inference could be calculated as the genotypes are partitioned into parents, crosses and their interactions. The mean squares of parents, crosses and parent $x$ crosses interaction were significant in all studied traits, except parent $\times$ crosses interaction of fruit firmness, indicating the presence of considerable differences among these genotypes. Therefore, it become statistically valid for the required diversity for the success of the planned crosses. The lines showed significant differences for all the traits, except the non-significant differences for titratable acidity. Also, the testers exhibited significant differences for all the traits, except total yield and number of locules. While, line $x$ tester interaction showed significant differences for all studied traits, except number of locules trait. These results are in agreement with those of Mondal et al (2009), Kansouh and Zakher (2011), Katkar et al (2012), Kalenahalli and Gowda (2013), Shankar et al (2013), Saeed et al (2014), Dagade et al (2015) and Khalil et al (2015) on tomato crop.

\section{2- Components of variance, heritability, components of genetic variance and proportional contribution:}

Genotypic and phenotypic variance $\left(\sigma^{2}{ }_{g}\right.$ and $\sigma_{p}^{2}$ ), heritability in broad sense $(B S H)$, genotypic and phenotypic coefficient of variance (G.C.V. \% and P.C.V. \%) and the ratio of G.C.V./P.C.V. are shown in Table 2 . Estimated $\sigma^{2}{ }_{g}$ vs $\sigma_{p}^{2}$ for the studied traits were: 177.13 vs 194.55 for fruit set, 26.105 vs 35.390 for total yield, 18.03 vs 24.35 for marketable yield, 763.38 vs 775.62 for average fruit weight, 0.012 vs 0.014 for fruit shape index, 6758.23 vs 7901.56 for fruit firmness, 0.90 vs 1.56 for number of locules, 0.106 vs 0.146 for TSS\%, 7.74 vs 8.46 for vitamin $C$ content and 0.013 vs 0.021 for titratable acidity. In this respect, all the studied traits showed low values of difference between phenotypic and genotypic variance, except number of locules and titratable acidity traits which led to a close correspondence varies between genotypic and phenotypic coefficient of variations (G.C.V and P.C.V \%). Also, the G.C.V./P.C.V. ratios for the studied traits showed high values. Estimates of BSH were high for all studied traits, except number of locules and titratable acidity traits, which were moderate. These results indicated more effect of genetic and less effect of environment on these traits.

Generally, the smaller values of differences between $\sigma_{p}^{2}$ and $\sigma_{g}^{2}$ indicated the low environmental effect on all studied character. Also, estimated G.C.V/P.C.V. ratios and $\mathrm{BSH}$ confirmed these results. So, the phenotypic values represented truly the genotypic values which indicated that the selection based on the phenotypic values will be effective for improvement of all studied traits. These results are partially agreed with Kansouh and Zakher (2011) and Salib (2012).

The data in Table 2 showed that lines gave variances higher than testers for the characters marketable yield, TSS $\%$ and vitamin C content, however, testers gave variances higher than lines for the characters fruit set, total yield, average fruit weight, fruit firmness, number of locules and titratable acidity, meanwhile, they are the same in the trait fruit shape. These results 


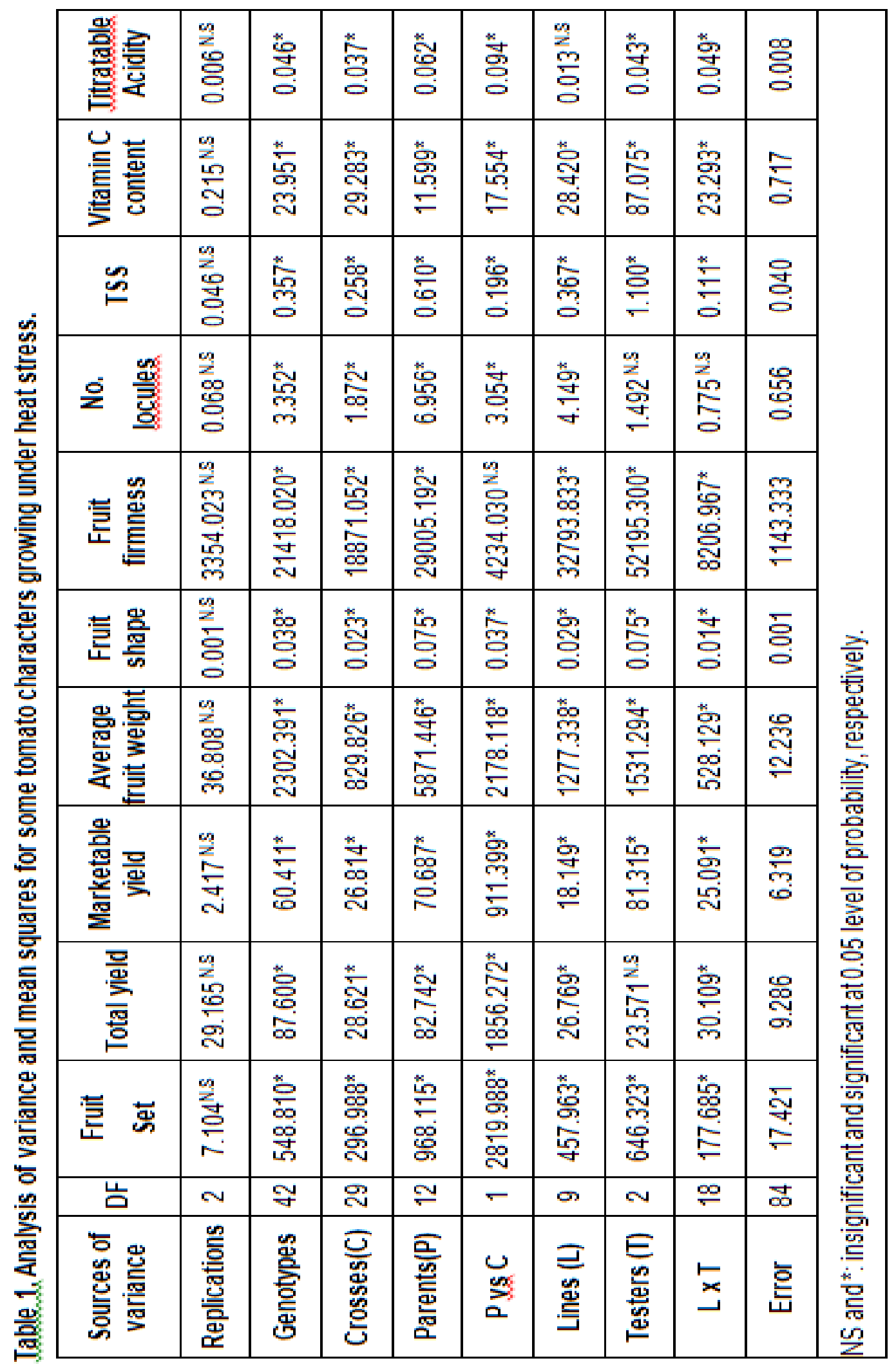




\begin{tabular}{|c|c|c|c|c|c|c|c|c|c|c|c|c|c|c|c|c|c|}
\hline 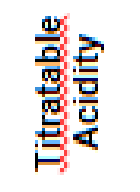 & & $\stackrel{m}{\circ}$ & চ্ণ & $\stackrel{\check{\circ}}{\circ}$ & 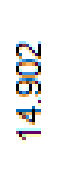 & $\frac{\check{N}}{\stackrel{\sigma}{\sigma}}$ & $\mid \begin{array}{l}\infty \\
⿱ \\
0\end{array}$ & & 훙 & 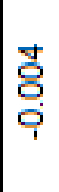 & 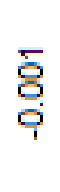 & 훙 & ปี & & $\stackrel{\Xi}{\stackrel{\circ}{-}}$ & Sু & $\begin{array}{l}\mathscr{8} \\
\stackrel{\circ}{\circ}\end{array}$ \\
\hline 㝕 & & $\underset{r}{\pi}$ & $\begin{array}{l}\infty \\
\stackrel{\infty}{*}\end{array}$ & హั & $\begin{array}{l}0.0 \\
\stackrel{6}{\circ} \\
\stackrel{0}{\circ}\end{array}$ & 오 & 号 & & $\frac{m}{i}$ & 茴 & $\bar{\sigma}$ & $\begin{array}{l}\text { Sू } \\
\text { ரे }\end{array}$ & 훙 & & 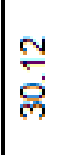 & 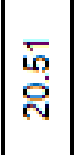 & ले \\
\hline$\stackrel{\tilde{n}}{\mathscr{H}}$ & & $\frac{8}{\circ}$ & $\frac{\varphi}{\frac{Q}{0}}$ & $\stackrel{m}{\stackrel{m}{0}}$ & 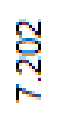 & $\underset{\infty}{\stackrel{y}{Z}}$ & $\begin{array}{l}30 \\
0 \\
0 \\
0 \\
0\end{array}$ & & 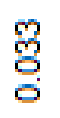 & ֻ̊ & 웅 & $\stackrel{\circ}{\circ}$ & 응 & & $\begin{array}{l}\text { 号 } \\
\text { J }\end{array}$ & $\begin{array}{l}\text { ले } \\
\text { ले } \\
\text { ले }\end{array}$ & 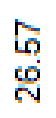 \\
\hline$\dot{2} \frac{\stackrel{6}{3}}{z}$ & & 용 & $\stackrel{\circ}{\stackrel{0}{6}}$ & 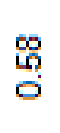 & 竞 & $\begin{array}{l}\text { i } \\
\text { o } \\
\text { N }\end{array}$ & $\begin{array}{l}\mathscr{0} \\
\stackrel{0}{\circ} \\
0\end{array}$ & & $\stackrel{\circ}{\circ}$ & ले. & $\stackrel{\circ}{\circ}$ & 缹 & $\stackrel{\circ}{\circ}$ & & $\begin{array}{l}2 \\
2 \\
0 \\
0\end{array}$ & 屌 & $\begin{array}{l}\overline{1} \\
\text { in } \\
\text { in }\end{array}$ \\
\hline 步点离 & & 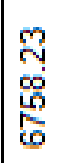 & 造 & $\begin{array}{l}\mathscr{\circ} \\
\stackrel{\circ}{\circ}\end{array}$ & \begin{tabular}{l}
$\circ$ \\
$\infty$ \\
0 \\
\hdashline
\end{tabular} & $\stackrel{\infty}{\infty}$ & 今̊ & $\underset{\mathrm{E}}{\stackrel{\Xi}{\mathrm{W}}}$ & 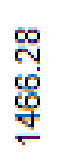 & 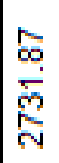 & $\begin{array}{l}\text { ळ } \\
\stackrel{\mathrm{g}}{\sigma}\end{array}$ & 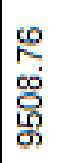 & $\stackrel{8}{\circ}$ & ‡ & $\begin{array}{l}\text { \% } \\
\text { ஸై }\end{array}$ & $\begin{array}{l}\text { \% } \\
\stackrel{\circ}{\circ} \\
\end{array}$ & $\begin{array}{l}\text { म } \\
\text { ì }\end{array}$ \\
\hline 壱㟧总 & 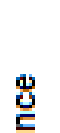 & 응 & $\stackrel{+}{\circ}$ & $\begin{array}{l}\mathscr{8} \\
\stackrel{0}{\circ}\end{array}$ & 守 & 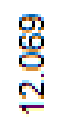 & 岁 & 总 & రั & 웅 & 8 & 훙 & $\stackrel{m}{\circ}$ & 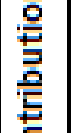 & 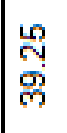 & 昌 & $\begin{array}{l}\text { त् } \\
\text { o }\end{array}$ \\
\hline 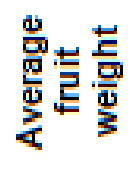 & 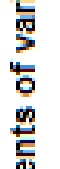 & 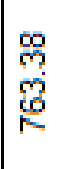 & $\begin{array}{l}\text { ญू } \\
\text { ผ̊ } \\
\text { है }\end{array}$ & 哭 & 只 & 岕 & \% & 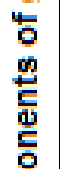 & 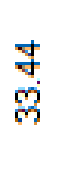 & 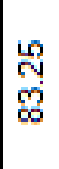 & $\begin{array}{l}\text { J్ } \\
\stackrel{\circ}{\circ}\end{array}$ & फ़ & $\stackrel{\circ}{\circ}$ & $\begin{array}{l}\frac{\mathrm{U}}{\mathrm{g}} \\
\mathrm{c} \\
\stackrel{\mathrm{o}}{\mathrm{E}} \\
\mathrm{t}\end{array}$ & $\begin{array}{l}E \\
\text { 守 }\end{array}$ & $\stackrel{\mathscr{m}}{\stackrel{M}{N}}$ & $\begin{array}{l}\text { 유 } \\
\text { ब्ल }\end{array}$ \\
\hline 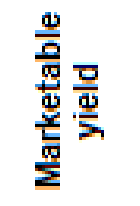 & 흘 & 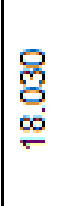 & 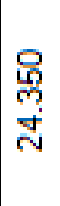 & $\frac{5}{5}$ & 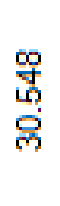 & $\begin{array}{l}5 \\
\text { फ़ } \\
\text { ê }\end{array}$ & $\begin{array}{l}\mathscr{0} \\
\mathscr{0} \\
0\end{array}$ & 통 & $\underset{\infty}{\stackrel{0}{\infty}}$ & $\begin{array}{l}\bar{E} \\
\dot{E} \\
0\end{array}$ & ช్ర & $\stackrel{\mathscr{8}}{=}$ & \% & & চ্் & 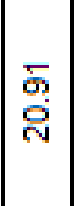 & 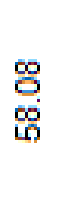 \\
\hline 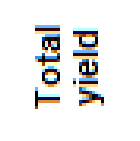 & & $\frac{20}{\circ}$ & 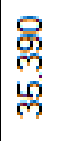 & 告 & $\begin{array}{l}3 \\
8 \\
0 \\
\infty \\
i\end{array}$ & 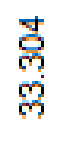 & $\begin{array}{l}\mathscr{0} \\
\mathscr{0} \\
0\end{array}$ & & 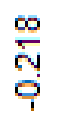 & 容 & 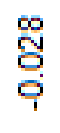 & 家 & 용 & & \% & 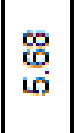 & 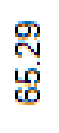 \\
\hline 志芯 & & $\stackrel{m}{\stackrel{m}{E}}$ & $\begin{array}{l}\text { 员 } \\
\text { 号 }\end{array}$ & চั & గ్రా & $\begin{array}{l}\stackrel{\leftrightarrow}{\circ} \\
\stackrel{N}{N}\end{array}$ & 岁 & & 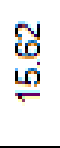 & 离 & $\stackrel{\text { N }}{\text { N }}$ & 官 & $\stackrel{\circ}{\circ}$ & & \begin{tabular}{l}
$\mathscr{0}$ \\
$\mathscr{8}$ \\
\multirow{5}{*}{}
\end{tabular} & 它 & $\frac{j}{\dot{m}}$ \\
\hline 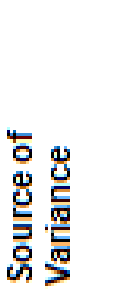 & & वा & : & $\begin{array}{l}\text { 工 } \\
\text { 品 }\end{array}$ & $\begin{array}{l}3 \\
ن \\
ن \\
ن\end{array}$ & $\begin{array}{l}2 \\
\text { > } \\
\dot{0}\end{array}$ & $\begin{array}{l}> \\
ن \\
ن \\
ن \\
ن \\
ن \\
ن\end{array}$ & & 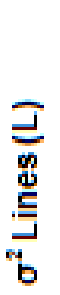 & 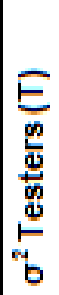 & 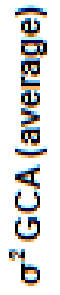 & 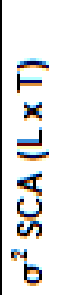 & 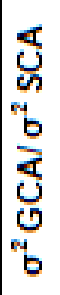 & & 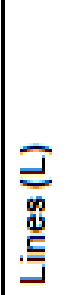 & 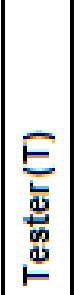 & $\stackrel{\vec{F}}{\stackrel{x}{2}}$ \\
\hline
\end{tabular}




\section{Combining ability and heterosis in tomato under high temperature conditions.}

indicated the importance of choice the parents. The results in Table 2 indicted that GCA and SCA variances showed wide range of variation for all studied characters. In all studied traits, SCA variances were greater than GCA variances and the ratio of $\sigma^{2} G C A / \sigma^{2} S C A$ were found less than unity $(<1)$. The higher magnitude of SCA variances indicates the preponderance of non additive gene actions over the additive ones for these characters. The prevalence of the non-additive variance suggesting heterosis breeding approach is effective way for improvement these traits. These results are in agreement with Mondal et al (2009), Kansouh and Zakher (2011), Katkar et al (2012), Kalenahalli and Gowda (2013), Shankar et al (2013), Saeed et al (2014), Dagade et al (2015) and Khalil et al (2015), who indicated the predominance of nonadditive gene actions for the characters fruit set, total yield, average fruit weight, fruit firmness, total soluble solids, ascorbic acid and titratable acidity.

The data of Table 2 indicated that testers had lower proportional contribution than lines and lines $x$ testers for all studied traits except TSS\% trait. Results also showed that lines were more important for productive for the traits fruit set $(47.86 \%)$, average fruits weight $(47.77 \%)$, fruit firmness $(53.93 \%)$, number of locules (68.79\%) and TSS\% $(44.07 \%)$ which revealed predominance influence for these traits. However, the contribution of maternal and paternal interaction (Line $x$ Tester) played higher important role higher than the individual contribution for the traits total yield $(65.294 \%)$, marketable yield $(58.081 \%)$ and vitamin C content (49.37).

\section{3- Mean performance and better- parent heterosis (Heterobeltiosis):}

Data obtained on performance of parents and their $F_{1}$ hybrids are presented in Table
3. Presented data showed significant differences for all studied traits among the evaluated genotypes.

The two testers $T_{1}$ and $T_{2}$ produced the highest significant fruit set percentage (86.69\% and $83.95 \%$, respectively) among all evaluated parents with non-significant differences between them. While, the line $L_{1}$ gave the lowest value (35.00\%). Regarding crosses, $L_{3} \times T_{3}$ and $L_{7} \times T_{1}$ gave the highest fruit set values $(80.37 \%$ and $79.84 \%$, respectively) with non-significant differences between them. With regard to heterosis, only 3 crosses out of the 30 evaluated ones $\left(L_{3} \times T_{3}, L_{4} \times T_{3}\right.$ and $\left.L_{6} \times T_{3}\right)$ exhibited significant positive heterosis over better parent ranging from $16.3 \%$ to $35.9 \%$.

For total yield, the line $L_{9}$ (18.014 ton) and tester $T_{1}$ gave maximum yield (17.131 ton) among all evaluated parents with nonsignificant differences between them. The hybrid $L_{2} \times T_{3}$ produced the highest total yield (26.759 ton) among all evaluated hybrids followed, respectively, by the hybrids $\mathrm{L}_{3} \times \mathrm{T}_{3}$ (25.981 ton) and $\mathrm{L}_{7} \times \mathrm{T}_{1}$ (25.744 ton) with non-significant differences between them. For heterosis, 13 out of the evaluated hybrids showed significant positive heterosis ranging from 32.7 to $99.0 \%$ for the crosses $L_{9} \times T_{1}$ and $L_{4} \times T_{3}$, respectively.

The data on marketable yield trait showed that the tester $T_{1}$ produced the maximum marketable yield (16.261 ton) among all evaluated parents, followed by the line $L_{9}$ (14.555 ton) with non-significant differences between them. For hybrids, the highest significant marketable yield was produced by the hybrid $L_{7} \times T_{1}$ (24.367 ton), followed by the hybrid $L_{10} \times T_{1}$ (22.539 ton) with non-significant differences between them. Concerning heterosis, 7 out of the 30 evaluated hybrids exhibited significant positive heterosis ranging from $21.4 \%$ to $71.0 \%$. 
Table 3. Mean performance and heterosis over better parent (BPH) for some economic characters of some tomato genotypes and their $F_{1}$ 's growing under heat stress.

\begin{tabular}{|c|c|c|c|c|c|c|c|c|c|c|}
\hline \multirow[b]{2}{*}{ Genotypes } & \multicolumn{2}{|c|}{ Fruit set (\%) } & \multicolumn{2}{|c|}{$\begin{array}{l}\text { Total yield } \\
\text { (ton/fed.) }\end{array}$} & \multicolumn{2}{|c|}{$\begin{array}{c}\text { Marketable } \\
\text { yield (ton/fed.) }\end{array}$} & \multicolumn{2}{|c|}{$\begin{array}{l}\text { Average fruit } \\
\text { weight (g) }\end{array}$} & \multicolumn{2}{|c|}{$\begin{array}{l}\text { Fruit shape } \\
\text { index }\end{array}$} \\
\hline & M & BPH & M & BPH & M & BPH & $\mathbf{M}$ & BPH & $\mathbf{M}$ & BPH \\
\hline $\mathrm{L}_{1}$ (Ent 2) & 35.00 & & 9.617 & & 8.600 & & 64.07 & & 0.90 & \\
\hline $\mathrm{L}_{2}$ (Ent 3) & 44.84 & & 14.981 & & 13.278 & & 208.27 & & 0.81 & \\
\hline $\mathrm{L}_{3}$ (Ent 5) & 38.93 & & 15.367 & & 13.000 & & 65.60 & & 1.08 & \\
\hline $\mathrm{L}_{4}$ (Ent 8) & 37.28 & & 5.833 & & 4.000 & & 65.63 & & 0.95 & \\
\hline $\mathrm{L}_{5}$ (Ent 9) & 44.15 & & 8.292 & & 4.333 & & 65.33 & & 0.95 & \\
\hline $\mathrm{L}_{6}$ (Ent 12) & 45.72 & & 4.628 & & 3.667 & & 52.10 & & 0.93 & \\
\hline $\mathrm{L}_{7}$ (Ent 17) & 62.96 & & 17.659 & & 13.547 & & 66.90 & & 0.89 & \\
\hline $\mathrm{L}_{8}$ (Ent 28) & 37.22 & & 3.129 & & 2.000 & & 97.77 & & 1.01 & \\
\hline$L_{9}$ (Ent 31) & 64.72 & & 18.014 & & 14.555 & & 109.07 & & 0.85 & \\
\hline $\mathrm{L}_{10}$ (Ent 37) & 71.15 & & 15.990 & & 12.000 & & 92.97 & & 0.94 & \\
\hline$T_{1}$ (TLB 111) & 86.69 & & 17.131 & & 16.261 & & 61.13 & & 1.42 & \\
\hline $\mathrm{T}_{2}$ (TLB 182-1) & 83.95 & & 15.120 & & 13.500 & & 57.47 & & 1.07 & \\
\hline $\mathrm{T}_{3}$ (99S-C-39) & 59.12 & & 11.541 & & 9.467 & & 25.10 & & 1.12 & \\
\hline$L_{1} \times T_{1}$ & 71.38 & $-17.7^{*}$ & 20.773 & 21.3 & 16.361 & 0.6 & 110.00 & $71.0^{*}$ & 0.88 & $-38.0^{*}$ \\
\hline$L_{1} \times T_{2}$ & 47.83 & $-43.0^{*}$ & 19.333 & 27.9 & 17.500 & 29.6 & 80.00 & 24.9 & 0.89 & $-16.8^{\star}$ \\
\hline$L_{1} \times T_{3}$ & 50.37 & $-14.8^{*}$ & 18.012 & $56.1^{*}$ & 14.986 & $58.3^{*}$ & 84.00 & 31.1 & 0.86 & $-23.2^{*}$ \\
\hline $\mathrm{L}_{2} \times \mathrm{T}_{1}$ & 63.62 & $-26.6^{*}$ & 19.869 & 16 & 16.375 & 0.7 & 111.47 & $-46.5^{\star}$ & 0.93 & $-34.5^{\star}$ \\
\hline$L_{2} \times T_{2}$ & 44.62 & $-46.9^{*}$ & 17.037 & 12.7 & 10.592 & -21.5 & 119.00 & $-42.9^{*}$ & 0.80 & $-25.2^{*}$ \\
\hline$L_{2} \times T_{3}$ & 63.24 & 7.0 & 26.759 & $78.6^{*}$ & 17.178 & 29.4 & 91.97 & $-55.8^{*}$ & 0.95 & $-15.2^{*}$ \\
\hline$L_{3} \times T_{1}$ & 76.90 & $-11.3^{*}$ & 19.716 & 15.1 & 15.389 & -5.4 & 107.40 & $63.7^{*}$ & 1.12 & $-21.2^{\star}$ \\
\hline$L_{3} \times T_{2}$ & 67.59 & $-19.5^{*}$ & 18.165 & 18.2 & 14.400 & 6.7 & 86.27 & $31.5^{*}$ & 0.94 & $-13.0^{\star}$ \\
\hline $\mathrm{L}_{3} \times \mathrm{T}_{3}$ & 80.37 & $35.9^{*}$ & 25.981 & $69.1^{*}$ & 17.643 & $35.7^{*}$ & 79.13 & 20.6 & 1.00 & $-10.7^{\star}$ \\
\hline $\mathrm{L}_{4} \times \mathrm{T}_{1}$ & 71.41 & $-17.6^{*}$ & 20.283 & 18.4 & 14.683 & -9.7 & 90.13 & $37.3^{*}$ & 0.95 & $-33.1^{*}$ \\
\hline$L_{4} \times T_{2}$ & 48.91 & $-41.7^{*}$ & 20.670 & $36.7^{*}$ & 17.259 & 27.8 & 89.00 & $35.6^{*}$ & 0.91 & $-15.0^{\star}$ \\
\hline $\mathrm{L}_{4} \times \mathrm{T}_{3}$ & 74.38 & $25.8^{*}$ & 22.971 & $99.0^{*}$ & 15.677 & $65.6^{*}$ & 82.47 & 25.7 & 1.01 & $-9.8^{*}$ \\
\hline$L_{5} \times T_{1}$ & 74.44 & $-14.1^{*}$ & 21.214 & 23.8 & 16.772 & 3.1 & 103.17 & $57.9^{*}$ & 0.95 & $-33.1^{*}$ \\
\hline$L_{5} \times T_{2}$ & 78.18 & -6.9 & 22.179 & $46.7^{*}$ & 16.179 & 19.8 & 104.27 & $59.6^{*}$ & 0.89 & $-16.8^{\star}$ \\
\hline$L_{5} \times T_{3}$ & 64.48 & 9.1 & 17.611 & $52.6^{*}$ & 13.111 & 38.5 & 69.83 & 6.9 & 0.97 & $-13.4^{*}$ \\
\hline $\mathrm{L}_{6} \times \mathrm{T}_{1}$ & 55.76 & $-35.7^{*}$ & 15.445 & -9.8 & 14.481 & -11.0 & 54.97 & -10.1 & 1.20 & $-15.5^{\star}$ \\
\hline $\mathrm{L}_{6} \times \mathrm{T}_{2}$ & 55.79 & $-33.5^{\star}$ & 19.375 & 28.1 & 14.350 & 6.3 & 75.73 & $31.8^{*}$ & 0.96 & $-10.3^{*}$ \\
\hline $\mathrm{L}_{6} \times \mathrm{T}_{3}$ & 68.75 & $16.3^{*}$ & 17.993 & $55.9^{*}$ & 13.993 & $47.8^{*}$ & 56.70 & 8.8 & 0.93 & $-17.0^{\star}$ \\
\hline$L_{7} \times T_{1}$ & 79.84 & -7.9 & 25.744 & $45.8^{*}$ & 24.367 & $49.9^{*}$ & 99.60 & $48.9^{*}$ & 0.97 & $-31.7^{\star}$ \\
\hline $\mathrm{L}_{7} \times \mathrm{T}_{2}$ & 75.71 & $-9.8^{*}$ & 22.056 & 24.9 & 17.233 & 27.2 & 86.87 & $29.9^{*}$ & 0.92 & $-14.0^{*}$ \\
\hline$L_{7} \times T_{3}$ & 68.90 & 9.4 & 18.211 & 3.1 & 13.055 & -3.6 & 81.30 & 21.5 & 0.90 & $-19.6^{\star}$ \\
\hline $\mathrm{L}_{8} \times \mathrm{T}_{1}$ & 69.84 & $-19.4^{*}$ & 19.737 & 15.2 & 13.307 & $\begin{array}{l}-18.2 \\
\end{array}$ & 72.33 & $-26.0^{*}$ & 0.93 & $-34.5^{\star}$ \\
\hline $\mathrm{L}_{8} \times \mathrm{T}_{2}$ & 68.89 & $-17.9^{*}$ & 18.809 & 24.4 & 13.910 & 3.0 & 89.67 & -8.3 & 0.89 & $-16.8^{\star}$ \\
\hline $\mathrm{L}_{8} \times \mathrm{T}_{3}$ & 63.14 & 6.8 & 18.151 & $57.3^{*}$ & 13.150 & 38.9 & 90.200 & -7.7 & 0.93 & $-17.0^{*}$ \\
\hline$L_{9} \times T_{1}$ & 57.25 & $-44.0^{*}$ & 23.910 & $32.7^{*}$ & 20.952 & $28.9^{*}$ & 63.200 & $-42.1^{*}$ & 1.21 & $-14.8^{\star}$ \\
\hline $\mathrm{L}_{9} \times \mathrm{T}_{2}$ & 52.09 & $-38.0^{*}$ & 15.989 & -11.2 & 12.700 & -12.8 & 105.300 & -3.5 & 0.91 & $-15.0^{\star}$ \\
\hline$L_{9} \times T_{3}$ & 66.89 & 3.4 & 15.574 & -13.5 & 11.648 & -20.0 & 68.900 & $-36.8^{*}$ & 0.98 & $-12.5^{\star}$ \\
\hline$L_{10} \times T_{1}$ & 66.67 & $-23.1^{*}$ & 24.960 & $45.7^{*}$ & 22.539 & $38.6^{*}$ & 112.833 & $21.4^{*}$ & 0.98 & $-31.0^{*}$ \\
\hline$L_{10} \times T_{2}$ & 57.89 & $-31.0^{*}$ & 20.486 & 28.1 & 15.829 & 17.3 & 86.933 & -6.5 & 1.01 & -5.6 \\
\hline $\mathrm{L}_{10} \times \mathrm{T}_{3}$ & 62.74 & $-11.8^{*}$ & 23.777 & $46.7^{*}$ & 13.870 & 15.6 & 95.867 & 3.1 & 0.94 & $-16.1^{*}$ \\
\hline LSD (5\%) & 6.89 & & 5.028 & & 4.148 & & 17.2 & & 0.10 & \\
\hline
\end{tabular}


Combining ability and heterosis in tomato under high temperature conditions.

Table 3. Continue.

\begin{tabular}{|c|c|c|c|c|c|c|c|c|c|c|}
\hline \multirow[b]{2}{*}{ Genotypes } & \multicolumn{2}{|c|}{$\begin{array}{c}\text { Fruit } \\
\text { firmness } \\
\left(\mathrm{g} / \mathrm{cm}^{2}\right)\end{array}$} & \multicolumn{2}{|c|}{ No. locules } & \multicolumn{2}{|c|}{ TSS $\%$} & \multicolumn{2}{|c|}{$\begin{array}{l}\text { Vitamin } \mathrm{C} \\
\text { content } \\
\text { ( } \mathrm{mg} / 100 \mathrm{~g} \\
\text { fresh fruit) }\end{array}$} & \multicolumn{2}{|c|}{$\begin{array}{c}\text { Titratable } \\
\text { Acidity (mg } \\
\text { citric } \\
\text { acid } / 100 \mathrm{~g} \\
\text { fresh fruit) }\end{array}$} \\
\hline & $\mathbf{M}$ & BPH & $\mathbf{M}$ & BPH & $\mathbf{M}$ & BPH & $\mathbf{M}$ & BPH & $\mathbf{M}$ & $\mathrm{BPH}$ \\
\hline $\mathrm{L}_{1}$ (Ent 2) & 325.0 & & 5.7 & & 4.7 & & 20.7 & & 0.62 & \\
\hline $\mathrm{L}_{2}$ (Ent 3) & 451.0 & & 7.1 & & 4.7 & & 16.5 & & 0.92 & \\
\hline $\mathrm{L}_{3}$ (Ent 5) & 642.0 & & 4.8 & & 4.4 & & 18.2 & & 0.72 & \\
\hline $\mathrm{L}_{4}$ (Ent 8) & 525.0 & & 4.2 & & 5.0 & & 20.0 & & 0.88 & \\
\hline $\mathrm{L}_{5}$ (Ent 9) & 412.0 & & 5.3 & & 4.2 & & 20.4 & & 0.65 & \\
\hline $\mathrm{L}_{6}$ (Ent 12) & 364.0 & & 3.3 & & 5.3 & & 19.6 & & 0.83 & \\
\hline $\mathrm{L}_{7}$ (Ent 17) & 563.0 & & 4.6 & & 4.2 & & 20.4 & & 1.04 & \\
\hline $\mathrm{L}_{8}$ (Ent 28) & 488.0 & & 5.4 & & 4.6 & & 18.4 & & 0.77 & \\
\hline $\mathrm{L}_{9}$ (Ent 31) & 635.0 & & 6.3 & & 4.1 & & 20.3 & & 0.73 & \\
\hline$L_{10}$ (Ent 37) & 542.0 & & 5.9 & & 4.1 & & 15.3 & & 0.71 & \\
\hline$T_{1}$ (TLB 111) & 483.0 & & 2.2 & & 4.4 & & 15.0 & & 0.59 & \\
\hline$T_{2}$ (TLB 182-1) & 405.0 & & 2.6 & & 4.5 & & 18.2 & & 0.84 & \\
\hline $\mathrm{T}_{3}$ (99S-C-39) & 414.0 & & 2.9 & & 5.5 & & 17.7 & & 1.01 & \\
\hline $\mathrm{L}_{1} \times \mathrm{T}_{1}$ & 432.0 & -10.6 & 5.0 & -12.2 & 4.3 & $-8.5^{\star}$ & 16.8 & $-18.9^{*}$ & 0.74 & 19.4 \\
\hline$L_{1} \times T_{2}$ & 437.0 & -7.9 & 4.5 & -21.1 & 4.8 & 2.1 & 17.4 & $-15.9^{*}$ & 0.83 & -1.2 \\
\hline$L_{1} \times T_{3}$ & 425.0 & 2.7 & 5.9 & 3.5 & 5.3 & -3.6 & 19.6 & -5.3 & 0.74 & $-26.7^{*}$ \\
\hline$L_{2} \times T_{1}$ & 618.0 & $28.0^{*}$ & 5.1 & $-28.2^{*}$ & 4.4 & $-6.4^{*}$ & 14.8 & $-10.3^{*}$ & 0.78 & -15.2 \\
\hline$L_{2} \times T_{2}$ & 520.0 & $15.3^{*}$ & 5.7 & $-19.7^{*}$ & 4.3 & $-8.5^{*}$ & 15.4 & $-15.4^{*}$ & 0.72 & $-21.7^{*}$ \\
\hline$L_{2} \times T_{3}$ & 422.0 & -6.4 & 4.4 & $-38.0^{*}$ & 4.7 & $-14.6^{*}$ & 24.2 & $36.7^{*}$ & 0.71 & $-29.7^{*}$ \\
\hline$L_{3} \times T_{1}$ & 575.0 & $-10.4^{*}$ & 3.5 & $-27.1^{*}$ & 4.4 & 0.0 & 19.1 & 5.0 & 0.70 & -2.8 \\
\hline $\mathrm{L}_{3} \times \mathrm{T}_{2}$ & 563.0 & $-12.3^{*}$ & 4.5 & -6.3 & 4.1 & $-8.8^{*}$ & 20.9 & $14.8^{*}$ & 0.74 & -11.9 \\
\hline$L_{3} \times T_{3}$ & 580.0 & $-9.7^{*}$ & 4.0 & -16.7 & 4.3 & $-21.8^{*}$ & 15.9 & $12.6^{*}$ & 0.79 & $-21.8^{*}$ \\
\hline $\mathrm{L}_{4} \times \mathrm{T}_{1}$ & 504.0 & -4.0 & 4.0 & -4.8 & 4.4 & $-12.0^{*}$ & 16.9 & $-15.5^{\star}$ & 0.74 & -15.9 \\
\hline $\mathrm{L}_{4} \times \mathrm{T}_{2}$ & 610.0 & $16.2^{*}$ & 3.9 & -7.1 & 4.6 & $-8.7^{*}$ & 20.6 & 3.0 & 0.84 & -4.6 \\
\hline $\mathrm{L}_{4} \times \mathrm{T}_{3}$ & 490.0 & -6.7 & 4.0 & -4.8 & 4.8 & $-12.7^{*}$ & 23.6 & $18.0^{*}$ & 0.74 & $-26.7^{*}$ \\
\hline$L_{5} \times T_{1}$ & 534.0 & 10.6 & 4.6 & $\begin{array}{l}-13.2 \\
\end{array}$ & 4.0 & $-9.1^{*}$ & 16.2 & $-20.6^{*}$ & 0.72 & 10.8 \\
\hline $\mathrm{L}_{5} \times \mathrm{T}_{2}$ & 445.0 & 8.0 & 4.3 & -18.9 & 4.4 & -2.2 & 15.5 & $-24.0^{*}$ & 0.77 & -8.3 \\
\hline$L_{5} \times T_{3}$ & 451.0 & 8.9 & 4.2 & -20.8 & 4.7 & $-14.6^{*}$ & 17.6 & $-13.7^{*}$ & 0.80 & $-20.8^{*}$ \\
\hline$L_{6} \times T_{1}$ & 544.0 & $12.6^{*}$ & 2.4 & -27.3 & 4.8 & $-9.4^{*}$ & 21.3 & $8.7^{*}$ & 0.70 & -15.7 \\
\hline $\mathrm{L}_{6} \times \mathrm{T}_{2}$ & 605.0 & $49.4^{*}$ & 3.4 & 3.0 & 4.8 & $-9.4^{*}$ & 21.6 & $10.2^{*}$ & 0.54 & $-35.7^{*}$ \\
\hline $\mathrm{L}_{6} \times \mathrm{T}_{3}$ & 525.0 & $26.8^{*}$ & 3.1 & -6.1 & 4.7 & $-14.6^{*}$ & 18.9 & -3.6 & 1.04 & 3.0 \\
\hline$L_{7} \times T_{1}$ & 515.0 & -8.5 & 4.3 & -6.5 & 4.0 & $-9.0^{*}$ & 18.4 & $-9.8^{*}$ & 0.58 & $-44.2^{*}$ \\
\hline $\mathrm{L}_{7} \times \mathrm{T}_{2}$ & 575.0 & 2.1 & 4.2 & -8.7 & 4.4 & -2.2 & 18.0 & $-11.8^{*}$ & 0.68 & $-34.6^{*}$ \\
\hline $\mathrm{L}_{7} \times \mathrm{T}_{3}$ & 427.0 & $-24.2^{*}$ & 3.7 & $\begin{array}{l}-19.6 \\
\end{array}$ & 4.7 & $-14.6^{*}$ & 15.7 & $-23.0^{*}$ & 0.92 & -11.5 \\
\hline $\mathrm{L}_{8} \times \mathrm{T}_{1}$ & 383.0 & $-21.5^{\star}$ & 4.6 & -14.8 & 4.5 & -2.2 & 10.5 & $-42.9^{*}$ & 0.84 & 9.1 \\
\hline $\mathrm{L}_{8} \times \mathrm{T}_{2}$ & 465.0 & -4.7 & 5.1 & -5.6 & 4.4 & -4.4 & 17.3 & -6.0 & 0.55 & $-34.5^{\star}$ \\
\hline $\mathrm{L}_{8} \times \mathrm{T}_{3}$ & 325.0 & $-33.4^{*}$ & 4.5 & -16.7 & 5.0 & $-9.1^{*}$ & 19.7 & 7.1 & 0.61 & $-39.6^{*}$ \\
\hline$L_{9} \times T_{1}$ & 399.0 & $-37.2^{*}$ & 2.8 & $-55.6^{*}$ & 4.3 & -2.3 & 12.0 & $-40.9^{*}$ & 0.70 & -4.1 \\
\hline$L_{9} \times T_{2}$ & 541.0 & $-14.8^{*}$ & 4.9 & $-22.2^{*}$ & 4.4 & -2.2 & 18.8 & $-7.4^{*}$ & 0.53 & $-36.9^{*}$ \\
\hline $\mathrm{L}_{9} \times \mathrm{T}_{3}$ & 336.0 & $-47.1^{*}$ & 3.8 & $-39.7^{*}$ & 4.7 & $-14.6^{*}$ & 18.8 & $-7.4^{*}$ & 0.78 & $-22.8^{*}$ \\
\hline $\mathrm{L}_{10} \times \mathrm{T}_{1}$ & 470.0 & $-13.3^{*}$ & 5.2 & -11.9 & 4.1 & $-6.8^{*}$ & 12.2 & $-20.3^{*}$ & 0.73 & 2.8 \\
\hline $\mathrm{L}_{10} \times \mathrm{T}_{2}$ & 565.0 & 4.2 & 5.1 & $\begin{array}{l}-13.6 \\
\end{array}$ & 4.3 & -4.4 & 16.8 & $-7.7^{*}$ & 0.83 & -1.2 \\
\hline $\mathrm{L}_{10} \times \mathrm{T}_{3}$ & 514.0 & -5.2 & 4.7 & -20.3 & 4.2 & $-23.6^{*}$ & 17.2 & -2.8 & 0.62 & $-38.6^{*}$ \\
\hline LSD (5\%) & 55.8 & & 1.3 & & 0.3 & & 1.4 & & 0.15 & \\
\hline
\end{tabular}


The highest average fruit weight was found in fruits of the line $L_{2}(208.27 \mathrm{~g})$ among all evaluated parents. Regarding hybrids, $L_{2} \times T_{2}(119.00 \mathrm{~g})$ gave the heaviest fruits followed by the hybrid $L_{10} \times T_{1}$ $(112.833 \mathrm{~g})$ with non-significant differences between them.

In case of fruit shape index trait, only the tester $T_{1}$ had oval fruits, meanwhile, the other testers $T_{2}$ and $T_{3}$ and all lines had round or oblate fruits, meanwhile, all evaluated hybrids gave round or oblate fruits. None of the 30 hybrids were superior for fruit shape index trait.

Fruit firmness of the evaluated parents ranged from $325.0 \mathrm{~g} / \mathrm{cm}^{2}\left(\mathrm{~L}_{1}\right)$ to $642.0 \mathrm{~g} / \mathrm{cm}^{2}$ $\left(L_{3}\right)$. The genotypes $L_{3}$ and $L_{9}$ had the highest fruit firmness among all evaluated parents, however, the hybrids $L_{2} \times T_{1}$ and $L_{4}$ $\times T_{2}$ gave the highest fruit firmness (618.0 and $610 \mathrm{~g} / \mathrm{cm}^{2}$, respectively) among all evaluated hybrids without significant differences between them. Six out of the 30 evaluated hybrids showed significant positive heterosis for fruit firmness ranged from $12.6 \%$ to $49.4 \%$.

The lines $L_{2}, L_{9}$ and $L_{10}$, significantly, had the highest number of locules among parents without significant differences between them. For hybrids, the hybrid $L_{1} \times$ $T_{3}$, significantly, had the highest number of locules (5.9) followed by $L_{2} \times T_{2}$ (5.7) without significant differences between them. None of the 30 hybrids were superior for number of locules trait.

For TSS\% trait, the highest TSS value of parents was detected in fruits of the tester $\mathrm{T}_{3}$ (5.5\%), meanwhile, the hybrid $L_{1} \times T_{3}$ had the highest TSS\% (5.3\%) followed by the hybrid $\mathrm{L}_{8} \times \mathrm{T}_{3}(5.0 \%)$ with significant differences between them. None of the 30 hybrids were superior for TSS\% trait.

Regarding ascorbic acid content trait, fruits of the line $L_{1}$ had the highest ascorbic acid content $(20.7 \mathrm{mg} / 100 \mathrm{~g}$ fresh fruit) among evaluated parents followed by the lines $L_{5}$ and $L_{7}(20.4 \mathrm{mg} / 100 \mathrm{~g}$ fresh fruit) without significant differences between them, however, the hybrid $L_{2} \times T_{3}$ had, significantly, the highest ascorbic acid content $(24.2 \mathrm{mg} / 100 \mathrm{~g}$ fresh fruit) among all evaluated hybrids followed by the hybrid $L_{4}$ $\times T_{3}(23.6 \mathrm{mg} / 100 \mathrm{~g}$ fresh fruit) without significant differences between them. Six out of the 30 evaluated hybrids showed significant positive heterosis for ascorbic acid content ranging from $8.7 \%$ to $36.7 \%$, respectively.

In case of titratable acidity trait, fruits of the line $L_{7}$ had, significantly, the highest titratable acidity $(1.04 \mathrm{mg} / 100 \mathrm{~g}$ fresh fruit) among evaluated parents, however the hybrid $L_{6} \times T_{3}$ had, significantly, the highest titratable acidity $(1.04 \mathrm{mg} / 100 \mathrm{~g}$ fresh fruit) among all evaluated hybrids followed by the hybrid $L_{7} \times T_{3}(0.92 \mathrm{mg} / 100 \mathrm{~g}$ fresh fruit) without significant differences between them. None of the 30 hybrids were superior for titratable acidity trait.

These results are partially in agreement with the findings of Mondal et al (2009), Shalaby (2012), Kalenahalli and Gowda (2013), Saeed et al (2014) and Khalil et al (2015) who found heterosis over better parent in tomato for the traits fruit set, total yield per plant average fruit weight, fruit firmness and TSS. However, heterosis was found absent for average fruit weight (Shalaby 2012), for fruit yield, TSS, and fruit firmness (Kalenahalli and Gowda 2013) and for fruit set trait (Khalil et al 2015).

\section{4- General Combining Ability Effects of Parents:}

Estimation of general combining ability (GCA) provides basic and important information for exploiting genetic potential of parents for development of superior lines. As expression of significant and high GCA effects of a parent line reflects the presence of favorable additive genes with additive 


\section{Combining ability and heterosis in tomato under high temperature conditions.}

genetic effects that leads to selection in early generations for developing widely adapted hybrids (Roy et al 2002). Estimation of GCA effects of lines and testers represented that no single line or tester exhibited good general combining ability for all the traits (Table 4). Among the lines, the highest values of GCA effects were shown by the line $L_{3}$ for fruit set percentage, fruit firmness and ascorbic acid content traits. The line $L_{10}$ gave the highest values for total yield and average fruit weight, while the line $\mathrm{L}_{7}$ had the highest GCA effects for marketable yield trait. The line $L_{9}$ gave the highest values for fruit shape index trait, while, the line $L_{1}$ had the highest number of locules per fruit and TSS traits. The line $L_{6}$ gave the highest values for ascorbic acid content trait. Similarly among the testers, $\mathrm{T}_{1}$ had the highest values for fruit set percentage, total yield, marketable yield, average fruit weight and fruit shape index. While, the line $T_{2}$ gave the highest values for fruit firmniss and number of locules/fruit. However, the highest values of GCA effects were shown by the line $T_{3}$ for TSS, ascorbic acid content and titratable acidity traits. According to these results, lines $L_{3}$ and $L_{7}$ and the tester $T_{1}$ showed maximum positive GCA effects for most of the important traits. So, these parents could be successfully used in future breeding programs.

\section{5- Specific Combining Ability Effects of Hybrids:}

The specific combining ability reveals the best cross combinations which can be useful for developing hybrids with high vigour for the traits. Significant superior SCA effects for all studied traits were not shown by a single hybrid. The data obtained in Table 5 indicated that the $F_{1}$ crosses $L_{1} \times T_{1}, L_{4} \times T_{3}$, $L_{5} \times T_{2}, L_{6} \times T_{3}, L_{7} \times T_{2}, L_{8} \times T_{2}$ and $L_{9} \times T_{3}$ achieved significant positive SCA effects for fruit set percentage. Only three crosses $\left(L_{2} \times\right.$ $T_{3}, L_{3} \times T_{3}$ and $L_{9} \times T_{1}$ ) showed significant SCA effects for total yield. Five crosses $\left(L_{2} \times\right.$ $\mathrm{T}_{3}, \mathrm{~L}_{3} \times \mathrm{T}_{3}, \mathrm{~L}_{7} \times \mathrm{T}_{1}, \mathrm{~L}_{9} \times \mathrm{T}_{1}$ and $\mathrm{L}_{10} \times \mathrm{T}_{1}$ ) showed significant SCA effects for marketable yield. Eleven hybrids exhibited significant SCA effects for heavy fruits and the cross $L_{9} \times T_{2}$ showed the highest significant value. The hybrid $L_{9} \times T_{1}$ showed the highest significant SCA effect for fruit shape index trait. Four crosses $\left(L_{2} \times T_{1}, L_{3} \times\right.$ $T_{3}, L_{5} \times T_{1}$ and $\left.L_{9} \times T_{2}\right)$ had significant positive SCA effects for fruit firmness trait. None of crosses showed significant SCA effect for number of locules/fruit trait. Only two crosses, viz., $\mathrm{L}_{1} \times \mathrm{T}_{3}$ and $\mathrm{L}_{3} \times \mathrm{T}_{1}$ showed significant positive SCA effects for TSS trait. Significant positive SCA effects were observed in nine crosses for ascorbic acid content trait and the hybrid $L_{2} \times T_{3}$ had the highest value. For titratable acidity trait, the SCA effects for $L_{6} \times T_{3}, L_{7} \times T_{3}, L_{8} \times T_{1}$ and $L_{10} \times T_{2}$ were significant and positive. Among all hybrids, $L_{2} \times T_{3}, L_{3} \times T_{3}$ and $L_{9} \times$ $\mathrm{T}_{1}$ exhibited significant SCA effects for both total and marketable yield characters. So, these hybrids can be used in future breeding program.

\section{CONCLUSION}

From this study, it can be concluded that the lines Ent 5 and Ent 17 and the tester TLB 111 showed maximum positive GCA effects for most of the important traits under heat stress. So, these parents could be successfully used in future breeding programs. Also, among all crosses, Ent $3 \times$ 99S-C-39, Ent $5 \times 99 S-C-39$ and Ent $31 \times$ TLB 111 exhibited significant SCA effects for both total and marketable yield characters under heat stress. So, these hybrids can be used in future breeding program. 


\begin{tabular}{|c|c|c|c|c|c|c|c|c|c|c|c|c|c|c|c|c|c|c|c|c|}
\hline$\frac{n}{b}$ & 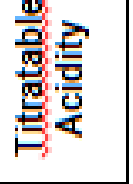 & & 㟧 & \% & 훙 & 웅 & ్ㅡㅇ & 峞 & Sُ & $\begin{array}{l}8 \\
8 \\
8 \\
0 \\
\end{array}$ & 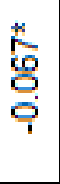 & \begin{tabular}{|l|} 
\\
0 \\
0
\end{tabular} & $\begin{array}{l}0 \\
8 \\
0 \\
0 \\
\end{array}$ & 产 & & 产 & 官 & 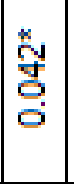 & ֻั & \\
\hline & 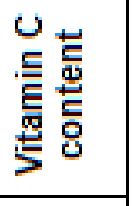 & & స̃ & ल & 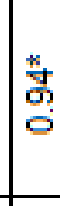 & 范 & ఫึَ & 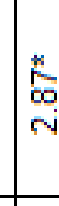 & $\stackrel{c}{0}$ & بै & ริ & 萬 & 总 & Tํํㅇ & & 苍 & 菅 & 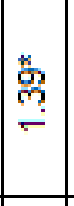 & స్ & \\
\hline & $\stackrel{\sim}{\mathscr{1}}$ & & ஜூ & 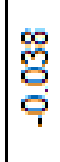 & స్ & $\frac{\mathscr{I}}{\stackrel{4}{\circ}}$ & 웅 & 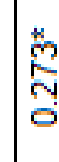 & ç & 它 & 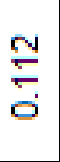 & 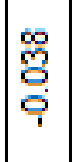 & 흘 & $\frac{\text { जे }}{5}$ & & 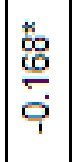 & $\begin{array}{l}\text { 웡 } \\
\text { 우 }\end{array}$ & 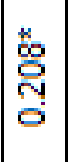 & 志 & \\
\hline & 을 $\frac{\text { dy }}{\vec{z}}$ & & 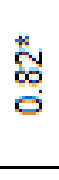 & 莡 & শั & लెִ & 응 & ले & 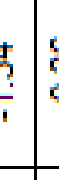 & 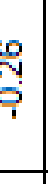 & ฯั & 辛 & 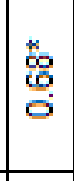 & 空 & & $\frac{n}{\grave{c}}$ & 뇸 & 竞 & స్త్రి & 离 \\
\hline & 苍营总 & $\bar{n}$ & 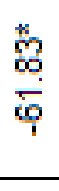 & 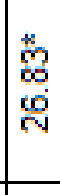 & $\begin{array}{l}\text { 苋 } \\
\text { के }\end{array}$ & 通 & $\begin{array}{l}0 \% \\
\stackrel{0}{\circ}\end{array}$ & 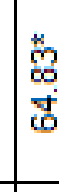 & : & 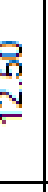 & $\frac{k}{\stackrel{*}{\circ}}$ & 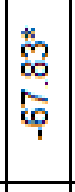 & $\frac{k}{\sigma}$ & $\begin{array}{l}\stackrel{\infty}{\mathfrak{N}} \\
\text { స̃ }\end{array}$ & & ָૈ & 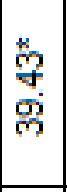 & 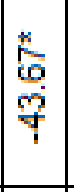 & $\stackrel{\text { g̊ }}{\stackrel{2}{ }}$ & $\begin{array}{l}\text { 讋 } \\
\text { 产 } \\
\text { 은 }\end{array}$ \\
\hline & 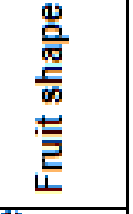 & 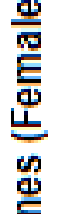 & 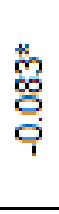 & 雚 & 苋 & 훙 & $\begin{array}{l}\text { هิ } \\
\text { ㅇ }\end{array}$ & 苞 & ç & '. & 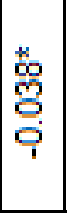 & 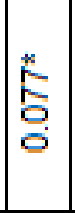 & ণ্ণ & : & 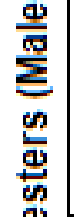 & 莒 & $\begin{array}{l}\text { * } \\
\text { 守 } \\
\text { ọ }\end{array}$ & 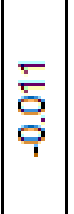 & $\stackrel{\square}{\circ}$ & 范 \\
\hline & 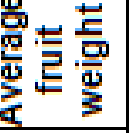 & ב & 苋 & 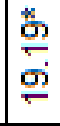 & 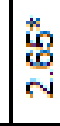 & $\frac{8}{7}$ & $\frac{*}{v}$ & 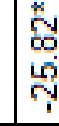 & $\begin{array}{l}y \\
0 \\
0 \\
\vdots \\
\vdots\end{array}$ & 今. & \begin{tabular}{l} 
ज̃ \\
\multirow{y}{*}{}
\end{tabular} & 产 & $\begin{array}{l}\text { ํㅜㅇ } \\
\text { 궁 } \\
\text { 으 }\end{array}$ & $\stackrel{\mathscr{্}}{\sim}$ & & $\stackrel{*}{*}$ & 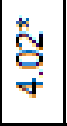 & 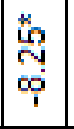 & $\stackrel{\text { న్ }}{=}$ & $\begin{array}{l}\text { 둥 } \\
\text { 응 } \\
0\end{array}$ \\
\hline & 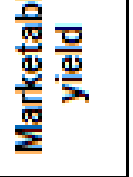 & & ஜூ & \%̆ & $\stackrel{\circ}{\circ}$ & స̃ & প্ণি & 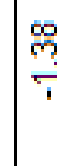 & 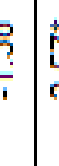 & مُ & $\frac{\mid}{\stackrel{*}{\circ}}$ & 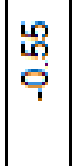 & $\stackrel{Q}{\mathscr{V}}$ & $\stackrel{g}{\dddot{g}}$ & & $\begin{array}{c}\stackrel{*}{*} \\
\stackrel{\infty}{\infty} \\
-\end{array}$ & $\begin{array}{l}\qquad 0 \\
0 \\
0 \\
\end{array}$ & $\stackrel{\text { స̂̀ }}{\stackrel{*}{*}}$ & ஜू & 思 \\
\hline & 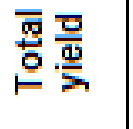 & & 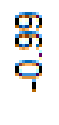 & $\begin{array}{l}0 \\
\infty \\
0\end{array}$ & \%ั & 通 & ه̊ & 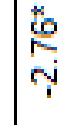 & is & 它 & 昌 & $\frac{\mathfrak{c}}{\infty}$ & $\frac{N}{i}$ & 结 & & $\begin{array}{c}\bar{\infty} \\
\dot{\infty}\end{array}$ & \begin{tabular}{|l|} 
\\
\\
\\
\end{tabular} & $\frac{\pi}{\dot{0}}$ & 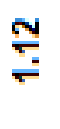 & 돔 \\
\hline & 觉 & & $\begin{array}{l}\text { 定 } \\
\text { o }\end{array}$ & $\begin{array}{l}* \\
k \\
r\end{array}$ & 웡 & O̊̊ & $\begin{array}{l}\text { * } \\
\text { v }\end{array}$ & $\begin{array}{l}* \\
\text { लू } \\
\text { क } \\
\text { पे }\end{array}$ & ?. & 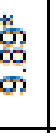 & 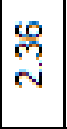 & 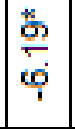 & $\begin{array}{l}\text { 员 } \\
\text { ণ }\end{array}$ & $\stackrel{\infty}{\sim}$ & & 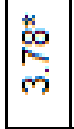 & 疍 & 운 & 悹 & 듬 \\
\hline & 屯ั & & 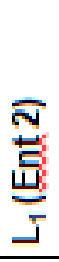 & 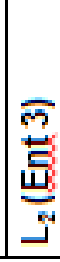 & 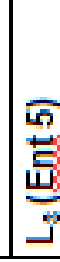 & $\stackrel{\infty}{\stackrel{\infty}{*}}$ & 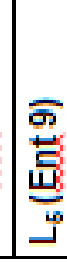 & 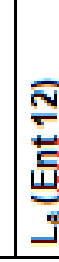 & 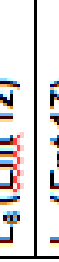 & $E$ & 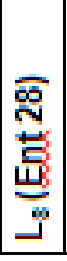 & 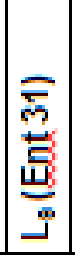 & 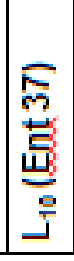 & 훙 & & 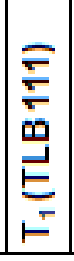 & 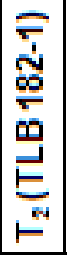 & 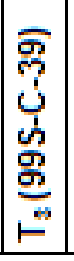 & 客 & 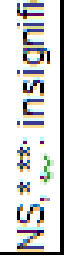 \\
\hline
\end{tabular}


Combining ability and heterosis in tomato under high temperature conditions.

Table 5. Estimation of specific combining ability (SCA) effects for some tomato characters growing under heat stress.

\begin{tabular}{|c|c|c|c|c|c|c|c|c|c|c|}
\hline Crosses & $\begin{array}{c}\text { Fruit } \\
\text { Set }\end{array}$ & $\begin{array}{l}\text { Total } \\
\text { yield }\end{array}$ & $\begin{array}{c}\text { Marketable } \\
\text { yield }\end{array}$ & $\begin{array}{c}\text { Average } \\
\text { fruit } \\
\text { weight }\end{array}$ & $\begin{array}{c}\text { Fruit } \\
\text { shape }\end{array}$ & $\begin{array}{c}\text { Fruit } \\
\text { firmness }\end{array}$ & $\begin{array}{c}\text { No. } \\
\text { locules }\end{array}$ & TSS & $\mid \begin{array}{c}\text { Vitamin } \\
\mathbf{C} \\
\text { content }\end{array}$ & $\begin{array}{c}\text { Titratable } \\
\text { Acidity }\end{array}$ \\
\hline & $11.07^{*}$ & & & & & -3.57 & 0.04 & & & .020 \\
\hline$\times T_{2}$ & -3.52 & 0.91 & & $-15.36^{*}$ & $0.056^{*}$ & -33.77 & -0.89 & 0.046 & $-108^{*}$ & 0.093 \\
\hline$x \mathrm{~T}$ & $-7.55^{\star}$ & -1.51 & -0.08 & 0.92 & -0.008 & 37.33 & 0.85 & $0.247^{*}$ & 0.29 & -0.073 \\
\hline $1 \times T$ & 2.68 & -2.16 & -0.21 & -0.24 & -0.021 & $93.77^{*}$ & 0.23 & 0.079 & $-1.45^{\star}$ & 0.055 \\
\hline $\mathrm{L}_{2} \times \mathrm{T}_{2}$ & $-7.36^{*}$ & -3.23 & $-3.47^{*}$ & $7.50^{*}$ & $-0.047^{*}$ & -39.43 & 0.37 & -0.149 & $-3.21^{*}$ & 0.011 \\
\hline $\mathrm{L}_{2} \times \mathrm{T}_{3}$ & 4.69 & $5.39^{*}$ & $3.68^{*}$ & $-7.26^{*}$ & $0.067^{*}$ & $-54.33^{*}$ & $-0.60^{*}$ & 0.069 & $4.66^{*}$ & -0.066 \\
\hline$L_{3} \times T_{1}$ & -1.84 & -2.38 & -2.29 & $12.24^{*}$ & 0.041 & -1.90 & -0.32 & $0.257^{*}$ & $2.37^{*}$ & -0.033 \\
\hline $\mathrm{L}_{3} \times \mathrm{T}_{2}$ & -2.18 & -2.17 & -0.76 & $-8.69^{*}$ & -0.034 & $-49.10^{*}$ & 0.26 & -0.088 & $1.77^{*}$ & 0.030 \\
\hline$L_{3} \times T_{3}$ & 4.02 & $4.55^{\star}$ & $3.05^{*}$ & -3.55 & -0.007 & $51.00^{*}$ & 0.06 & -0.169 & $-4.13^{*}$ & 0.003 \\
\hline $\mathrm{L}_{4} \times \mathrm{T}_{1}$ & 2.73 & -1.83 & $-3.06^{*}$ & -1.29 & $-0.060^{*}$ & -34.90 & 0.19 & -0.054 & $-1.53^{*}$ & -0.025 \\
\hline $\mathrm{L}_{4} \times \mathrm{T}_{2}$ & $-10.81^{*}$ & 0.31 & 2.04 & -2.22 & 0.001 & 35.90 & -0.30 & 0.051 & -0.29 & 0.101 \\
\hline $\mathrm{L}_{4} \times \mathrm{T}_{3}$ & $8.08^{*}$ & 1.52 & 1.02 & 3.52 & $0.060^{*}$ & -1.00 & 0.10 & 0.003 & $1.82^{*}$ & -0.076 \\
\hline$L_{5} \times T_{1}$ & -1.71 & 0.07 & -0.46 & $6.52^{*}$ & -0.044 & $53.10^{*}$ & 0.38 & -0.198 & $1.67^{*}$ & -0.033 \\
\hline$L_{5} \times T_{2}$ & $10.99^{*}$ & 2.79 & 1.48 & $7.82^{*}$ & 0.001 & $-71.10^{*}$ & -0.27 & 0.073 & $-1.45^{\star}$ & 0.037 \\
\hline$L_{5} \times T_{3}$ & $-9.28^{*}$ & -2.87 & -1.02 & $-14.34^{*}$ & 0.044 & 18.00 & -0.11 & 0.125 & -0.22 & -0.003 \\
\hline$L_{6} \times T_{1}$ & $-8.12^{*}$ & -2.96 & -1.67 & $-11.72^{*}$ & $0.118^{*}$ & -18.23 & -0.40 & 0.202 & $2.59^{*}$ & -0.051 \\
\hline$L_{6} \times T_{2}$ & 0.87 & 2.72 & 0.73 & $9.24^{*}$ & -0.031 & 7.57 & 0.15 & 0.073 & 0.51 & $-0.191^{*}$ \\
\hline $\mathrm{L}_{6} \times \mathrm{T}_{3}$ & $7.26^{*}$ & 0.24 & & 2.48 & $-0.088^{*}$ & 10.67 & 0.25 & $-0.275^{*}$ & $-3.10^{*}$ & $0.242^{*}$ \\
\hline$L_{7} \times T_{1}$ & 1.24 & 2.94 & & & -0.016 & 5.10 & 0.38 & -0.187 & $2.95^{*}$ & $-0.134^{\star}$ \\
\hline$L_{7} \times T_{2}$ & $6.07^{*}$ & 1.00 & -0.33 & $-6.37^{*}$ & 0.034 & 29.90 & -0.11 & 0.084 & 0.12 & -0.018 \\
\hline$L_{7} \times T_{3}$ & $-7.31^{*}$ & $-3.94^{*}$ & $-3.94^{*}$ & 0.27 & -0.017 & -35.00 & -0.27 & 0.103 & $-3.07^{*}$ & $0.152^{*}$ \\
\hline $\mathrm{L}_{8} \times \mathrm{T}_{1}$ & -1.23 & 0.03 & -2.02 & $-15.96^{*}$ & -0.041 & -12.23 & 0.00 & 0.029 & $-3.44^{*}$ & $0.185^{\star}$ \\
\hline $\mathrm{L}_{8} \times \mathrm{T}_{2}$ & $6.78^{*}$ & 0.86 & & 58 & 0.018 & 34.57 & 0.15 & -0.166 & 0.96 & -0.086 \\
\hline$L_{8} \times T_{3}$ & $-5.55^{\star}$ & -0.89 & 0.91 & $14.38^{*}$ & 0.023 & -22.33 & -0.15 & 0.136 & $2.49^{*}$ & -0.099 \\
\hline$L_{9} \times T_{1}$ & $-5.27^{*}$ & $4.61^{*}$ & $3.98^{*}$ & $-20.16^{*}$ & $0.122^{*}$ & -30.57 & -0.86 & 0.046 & $-2.64^{*}$ & 0.040 \\
\hline$L_{9} \times T_{2}$ & -1.47 & -1.55 & -1.75 & $22.14^{*}$ & -0.076 & $76.23^{*}$ & 0.78 & -0.049 & $1.77^{*}$ & $-0.110^{*}$ \\
\hline$L_{9} \times T_{3}$ & $6.75^{\star}$ & -3.06 & -2.23 & -1.98 & $-0.046^{*}$ & -45.67 & 0.08 & 0.003 & 0.87 & 0.070 \\
\hline$L_{10} \times T_{1}$ & 0.45 & 1.08 & $3.25^{\star}$ & $10.06^{*}$ & $-0.051^{*}$ & $-50.57^{*}$ & 0.35 & 0.118 & $-1.31^{*}$ & 0.017 \\
\hline $\mathrm{L}_{10} \times \mathrm{T}_{2}$ & 0.64 & -1.64 & -0.93 & $-15.64^{*}$ & $0.078^{*}$ & 9.23 & -0.14 & 0.123 & 0.91 & $0.133^{*}$ \\
\hline$L_{10} \times T_{3}$ & -1.09 & 0.56 & -2.32 & $5.57^{\star}$ & -0.027 & 41.33 & -0.21 & $-0.242^{*}$ & & $-0.150^{*}$ \\
\hline LSD 5\% & 4.87 & 3.56 & 2.93 & 4.08 & 0.045 & 39.45 & 0.95 & 0.232 & 0.99 & 0.105 \\
\hline
\end{tabular}

Note: Ent $2\left(L_{1}\right)$, Ent $3\left(L_{2}\right)$, Ent $5\left(L_{3}\right)$, Ent $8\left(L_{4}\right)$, Ent $9\left(L_{5}\right)$, Ent $12\left(L_{6}\right)$, Ent $17\left(L_{7}\right)$, Ent $28\left(L_{8}\right)$, Ent 31 $\left(\mathrm{L}_{9}\right)$, Ent $37\left(\mathrm{~L}_{10}\right)$, TLB $111\left(\mathrm{~T}_{1}\right)$, TLB 182-1 $\left(\mathrm{T}_{2}\right)$ and 99S-C-39 $\left(\mathrm{T}_{3}\right)$ 


\section{REFERENCES}

Abo-Hamda, E. M. E. H. (2004). Genetic studies on tomato male sterility. M.Sc. Thesis, Fac. Agric., Cairo Univ., Egypt, $97 \mathrm{p}$.

Berry, S. Z., M. Rafique and U. D. Din (1988). Effect of high temperature on fruit set in tomato cultivars and selected germplasm. Hort. Sci. 23: 606-608.

Dagade, S. B., L. K. Dhaduk, K. Hariprasanna, D. R. Mehata, V. M. Bhatt and A. V. Barad (2015). Parent offspring relations of nutritional quality traits in $8 x$ 8 partial diallel cross of fresh tomatoes. Inter. J. Appl. Bio. and Pharmaceutical Tech. 6 (2): 45-55.

El-Ahmadi, A. B. and M. A. Stevens (1979). Genetics of high temperature fruit set in the tomato. J. Amer. society hort. sci. 104: 691-696.

Griffing, B. (1956). Concept of general and specific combining ability in relation to diallel system. Aust. J. Biol. Sci., 9, 463493.

Kalenahalli, Y. and P. H. R. Gowda (2013). Line $\times$ tester analysis in tomato (Solanum lycopersicum L.): identification of superior parents for fruit quality and yieldattributing traits. Inter. J. Plant Breed. 7 (1): 50-54.

Kansouh, A. M. and A. G. Zakher (2011). Gene action and combining ability in tomato (Lycopersicon esculentum MILL.) by line $x$ tester analysis. J. Plant Production, Mansoura Univ. 2 (2): 213 227

Katkar, G. D., O. Sridevi, P. M. Salimath and S. P. Patil (2012). Combining ability analysis for yield, its contributing characters and fruit quality parameters of exotic tomato (Lycopersicon esculentum Mill.) breeding lines. Electronic J. Plant Breed. 3 (3): 908-915.

Kempthorne (1957). An Introduction to Genetic Statistics, John Wiley and Sons. Inc., New York, pp 458-471.

Khalil, E. M. E. A., A. A. Farrag, A. A. Kheder and H. M. Mazyad (2015).
Tomato breeding for heat and tomato yellow leaf curl virus (TYLCV) tolerance. Egypt. J. Appl. Sci. 30 (9): 556-579.

Kumar, R., K. Srivastava, N. P. Singh, N. K. Vasistha, R. K. Singh and M. K. Singh (2013). Combining ability analysis for yield and quality traits in tomato (Solanum lycopersicum L.). J. Agric. Sci. 5 (2): 213-218.

Metwally, E. I., G. El-Fadly and A. Mazrouh (1988). Inheritance of fruit set under heat stress conditions in tomato. Proc. 2nd Hort. Sci. conf. Tanta Univ. 521-530.

Mondal, C., S. Sarkar and P. Hazra (2009). Line $\times$ tester analysis of combining ability in tomato (Lycopersicon esculentum Mill.). J. Crop and Weed 5 (1): 53-57.

Saeed, A., N. Hasan, A. Shakeel, M. F. Saleem, N. H. Khan, K. Ziaf, R. A. M. Khan and N. Saeed (2014). Genetic analysis to find suitable parents for development of tomato hybrids. Researcher 6 (6): 77-82.

Salib, F. S. (2012). General and specific combining ability and genetic behavior of yield and some fruit characters of tomato under open field condition. Egypt . J. Appl. Sci. 27 (6):267-282.

Shalaby, T. A. (2012). Line $\times$ tester analysis for combining ability and heterosis in tomato under late summer season conditions. J. Plant Production, Mansoura Univ., 3 (11): 2857 - 2865.

Shankar, A., R. V. S. K. Reddy, M. Sujatha and M. Pratap (2013). Combining ability and gene action studies for yield and yield contributing traits in tomato (Solanum lycopersicum L.). Helix 6: 431435.

Sinha, S. K. and R. Khanna (1975). Physiological, biological and genetic basis of heterosis. Advances in Agronomy 27 (1): 123-174.

Sprague, G. F. and L. A. Tatum (1942). General vs. specific combining ability in single cross of corn. J. American Soc. Agron., 34: 923-32. 


\section{القدرة على التآلف وقوة الهجين فى الطماطم تحت ظروف الحرارة المرتفعة}

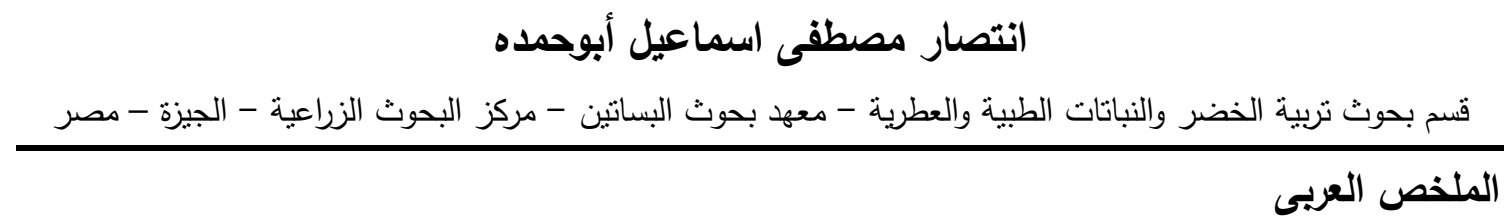

أجريت هذه الدراسة بمحافظة القليوبية خلال عامى 2014 ، 2015 وذلك بهدف استتباط بعض هجن من الطماطم المبشرة للمحصول العالى وصفات الجودة تحت ظروف الحرارة المرتفعة فى مصر بإستخدام طريقة التهجين القمى. أظهرت الدراسة وجود نطابق إلى حد كبير بين قيم كل من التباين الوراثى مع التباين الكلى وبين معامل الاختلاف الوراثى مع معامل

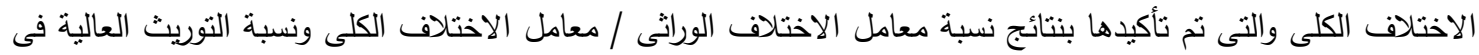
كل الصفات المدروسة وهى نسبة العقد ، والمحصول الكلى ، والمحصول القابل للنسويق ، ومتوسط وزن الثمرة ، وشكل الثمرة

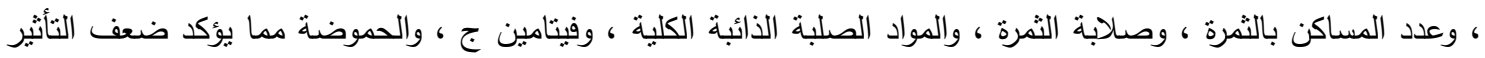

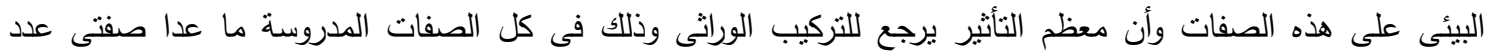

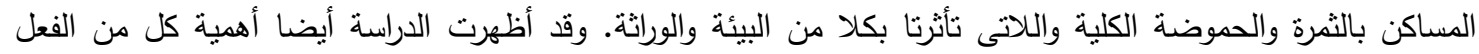

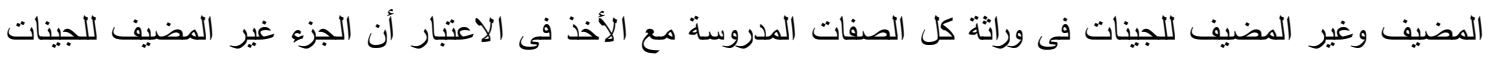

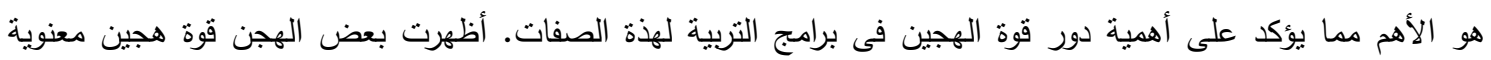

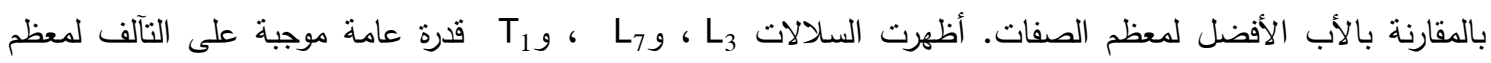
الصفات مما يؤكد امكانية استخدامها كآباء في برامج التربية. و قد أظهرت الهجن

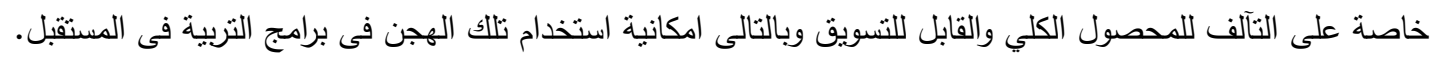


